\title{
UNICORE Summit 2008
}

The UNICORE grid technology provides a seamless, secure and intuitive access to distributed grid resources. UNICORE is a full-grown and well-tested grid middleware system, which today is used in daily production worldwide. Beyond this production usage, the UNICORE technology serves as a solid basis in many European and International projects. In order to foster these ongoing developments, UNICORE is available as open source under BSD licence at http://www.unicore.eu.

The UNICORE Summit is a unique opportunity for grid users, developers, administrators, researchers and service providers to meet. The First UNICORE Summit was held in conjunction with "Grids@work - 2nd Grid Plugtests,” October 11 - 12, 2005 in Sophia Antipolis, France. In 2006 the style of the UNICORE Summit was changed by establishing a Program Committee and publishing a Call for Papers. The UNICORE Summit 2006 was held in conjunction with the Euro-Par 2006 conference in Dresden, Germany, August $30-31,2006$. The proceedings are available as LNCS volume 4375. The UNICORE Summit 2007 was held in conjunction with the Euro-Par 2007 conference in Rennes, France, on August 28, 2008. The proceedings are available as LNCS volume 4854.

In 2008 the UNICORE Summit was held again in conjunction with the Euro-Par conference, this time in Las Palmas de Gran Canaria, Spain, on August 26.

We would like to thank the Program Committee members Agnes Ansari, Rosa Badia, Thomas Fahringer, Donal Fellows, Anton Frank, Edgar Gabriel, Alfred Geiger, Frerik Hedman, Odej Kao, Paolo Malfetti, Ralf Ratering, Mathilde Romberg, Bernd Schuller, Dave Snelling, Thomas Soddemann, Stefan Wesner and Ramin Yahyapour for their excellent job. Special thanks go to Max Berger and Kassian Plankensteiner for providing additional reviews.

Finally, we would like to thank all authors for their submissions, camera-ready versions and presentations at the UNICORE Summit 2008 in Las Palmas de Gran Canaria as well as Emilio Benfenati for giving the opening talk.

Most likely, the next UNICORE Summit will again take place in conjunction with the Euro-Par conference. More information can be found at http://www.unicore.eu/summit. We are looking forward to the next UNICORE Summit ! 